\title{
ESTUDO DE REGENERAÇÃO DE CATALISADOR Cu-Co-Al PARA A PRODUÇÃO DE HIDROGÊNIO A PARTIR DO GÁS NATURAL
}

\author{
F.M. BERNDT ${ }^{1}$, O.W. PEREZ LOPEZ ${ }^{1}$ \\ ${ }^{1}$ Universidade Federal do Rio Grande do Sul, Departamento de Engenharia Química \\ E-mail para contato: perez@enq.ufrgs.br
}

\begin{abstract}
RESUMO - Neste trabalho foram estudadas as condições de regeneração de catalisador de $\mathrm{Cu}-\mathrm{Co}-\mathrm{Al}$ para a produção de hidrogênio por decomposição catalítica do metano. As reações de decomposição de metano e a regeneração foram conduzidas na temperatura de $500^{\circ} \mathrm{C}$, sendo que foram avaliados diferentes tempos de regeneração assim como diferentes concentrações de oxigênio na mistura oxidante. As reações foram conduzidas num reator tubular acoplado a um forno elétrico e cromatógrafo gasoso em linha. Para os ensaios foi utilizada uma amostra de catalisador $\mathrm{Cu}-\mathrm{Co}-\mathrm{Al} \mathrm{com}$ granulometria média de $0,5 \mathrm{~mm}$. O carbono formado foi caracterizado através das análises de oxidação a temperatura programada (TPO). Os testes realizados com ar puro e tempo fixo de 10 minutos foram os que apresentaram melhor desempenho do catalisador. A análise termogravimétrica feita no catalisador com carbono depositado indicou um tempo adequado para a oxidação do carbono entre 10 e 15 minutos. As análises de TPO não apresentaram diferença significativa quanto à estrutura do carbono depositado durante a reação.
\end{abstract}

\section{INTRODUÇÃO}

Com a demanda cada vez maior por fontes energéticas menos poluentes, o hidrogênio aparece como uma promissora alternativa, pois além de possuir elevado poder energético, sua combustão não gera poluentes, apenas água.

Industrialmente, grande parte do hidrogênio utilizado é produzida pelo processo de reforma a vapor do gás natural (Armor, 1999; Holladay et al, 2009). No entanto, este processo produz monóxido de carbono e hidrogênio, mistura conhecida como gás de síntese, utilizado na indústria do metanol, entre outras. A obtenção de hidrogênio de alta pureza a partir do gás de síntese é um processo de custo muito elevado, sendo oportunas, portanto, rotas que produzam hidrogênio de elevada pureza (Armor, 1999).

Neste contexto a reação de decomposição catalítica do metano surge como uma interessante alternativa, pois o hidrogênio é produzido na forma pura, livre de óxidos de carbono (Hermes, 2010).

Nesta reação forma-se também, carbono sólido, que em condições especiais pode se depositar 


\section{9 a 22 de outubro de 2014 \\ Florianópolis/SC}

na forma de nanotubos, materiais muito estudados atualmente devido a suas excelentes propriedades mecânicas e elétricas (Avdeeva et al, 1999).

Devido a sua grande disponibilidade, o metano é uma matéria-prima de custo relativamente baixo, tendo como principal fonte o gás natural. Para que haja a quebra das ligações $\mathrm{C}-\mathrm{H}$ são necessárias temperaturas na faixa de $1200^{\circ} \mathrm{C}$. Nesse sentido, o uso de catalisadores reduz significativamente a temperatura de decomposição. Os metais mais ativos para este tipo de reação são os de transição como níquel, cobalto e ferro (Konieczny et al, 2008), podendo ser preparados sobre um suporte, como alumina ou sílica, ou coprecipitados com outros metais, como o alumínio, por exemplo. O cobalto é um metal bastante estudado na decomposição do metano, sendo usado tanto na forma pura quanto combinado a outros elementos. A combinação deste metal com um metal trivalente, no caso o alumínio, leva à formação de óxidos mistos $\left(\mathrm{CoAl}_{2} \mathrm{O}_{4}\right)$, o que confere alta estabilidade térmica ao catalisador, além de promover uma maior dispersão das partículas metálicas (Pérez-Ramirez et al, 2001).

O maior problema na decomposição do metano para a produção de hidrogênio é o entupimento do leito devido à deposição de carbono, exigindo a regeneração periódica do catalisador. Desta forma, ciclos de ativação, reação e regeneração são frequentemente usados neste tipo de processo (PerezLopez e Senger, 2004).

O objetivo deste trabalho é estudar a regeneração de um catalisador à base de $\mathrm{Cu}-\mathrm{Co}-\mathrm{Al}$ na decomposição do metano. A utilização, do catalisador $\mathrm{Cu}-\mathrm{Co}-\mathrm{Al}$ foi baseada em resultados obtidos em trabalhos anteriormente realizados (Escobar, 2012 e Hermes, 2010). Nestes trabalhos verificou-se que a substituição parcial de cobalto por cobre diminui drasticamente a temperatura de redução (ativação) do catalisador, sendo possível a redução do mesmo apenas com a corrente de alimentação do processo (metano e nitrogênio). Desta forma, a etapa de ativação é eliminada e o processo de produção de hidrogênio com catalisador $\mathrm{Cu}-\mathrm{Co}-\mathrm{Al}$ envolve apenas as etapas de reação e regeneração.

\section{EXPERIMENTAL}

\subsection{Preparação do Catalisador}

$\mathrm{O}$ catalisador utilizado neste trabalho foi à base de $\mathrm{Cu}$, Co e $\mathrm{Al}$ na proporção de 3:67:30 $\left(\mathrm{Cu}_{3} \mathrm{Co}_{67} \mathrm{Al}_{30}\right)$, sendo preparado pelo método de coprecipitação. A solução dos metais na forma de nitratos hidratados foi misturada lentamente com uma solução alcalina de carbonato de sódio. A reação de coprecipitação foi realizada em um reator CSTR de vidro, encamisado, sob agitação contínua nas seguintes condições: temperatura igual a $50^{\circ} \mathrm{C}$, controlada por um banho termostatizado e pH igual a 8,0 $\pm 0,1$. O pH da solução foi monitorado por um eletrodo de $\mathrm{pH}$ e controlado através da manipulação da vazão da solução alcalina. Após a precipitação, ocorreu a cristalização do produto por 1 hora também à temperatura de $50^{\circ} \mathrm{C}$. A seguir efetuou-se a filtração a vácuo e a lavagem do sólido utilizando-se um volume de água deionizada de 8 litros. Após, o material foi seco em estufa a $80^{\circ} \mathrm{C}$ por 24 horas para então ser moído e peneirado, sendo utilizado o material retido entre as peneiras de 32 e 42 mesh. O material selecionado foi calcinado na temperatura de $600^{\circ} \mathrm{C}$ durante 6 horas e sob um fluxo contínuo de ar na vazão de $50 \mathrm{~mL} / \mathrm{min}$. 


\section{9 a 22 de outubro de 2014 \\ Florianópolis/SC}

\subsection{Testes de Atividade do Catalisador}

Os ensaios catalíticos foram realizados em um reator tubular de vidro, com $370 \mathrm{~mm}$ de comprimento e $9 \mathrm{~mm}$ de diâmetro interno. A temperatura do leito foi medida por um termopar tipo $\mathrm{K}$ inserido na altura correspondente ao centro do leito catalítico, sendo controlada por um controlador digital de temperatura. A vazão dos gases foi estabelecida por controladores digitais de vazão mássica. $\mathrm{O}$ teste consistiu em aquecer o sistema até a temperatura previamente estipulada, em uma taxa de aquecimento de $10^{\circ} \mathrm{C}$ por minuto, mediante uma vazão de $90 \mathrm{~mL} / \mathrm{min}$ de nitrogênio e 10 $\mathrm{mL} / \mathrm{min}$ de metano. Após a estabilização da temperatura, o ensaio foi conduzido até a pressão no reator atingir 1 bar manométrico devido ao depósito de carbono no leito ou quando a queda da atividade foi superior a $50 \%$ da atividade inicial. A seguir a regeneração do catalisador foi efetuada. Após finalizada a regeneração, metano foi alimentado ao reator. Este procedimento foi executado sucessivamente num total de 4 ciclos de reação/regeneração. A massa de catalisador utilizada foi de $0,1 \mathrm{~g}$. Os produtos e o reagente não convertido foram analisados em linha através de um cromatógrafo gasoso usando nitrogênio como gás de arraste e detector de condutividade térmica.

\subsection{Regeneração do Catalisador}

Para a oxidação do carbono depositado sobre o catalisador foram utilizados ar sintético puro ou ar combinado com nitrogênio sob diferentes proporções. A temperatura de regeneração foi a mesma adotada na reação de decomposição catalítica. Nos testes preliminares as regenerações foram efetuadas em tempos fixos de 30 minutos de passagem do agente oxidante. Em seguida testes com tempos variáveis de regeneração forma utilizados. Para os testes finais utilizou-se tempo fixo de 10 minutos sob diferentes proporções de oxigênio na mistura oxidante.

\subsection{Oxidação à temperatura programada (TPO-DTA)}

Para investigar a formação de material carbonáceo sobre as partículas do catalisador e consequentemente, a tendência à desativação por deposição de coque, foram feitas análises de TPODTA em termobalança. A massa de catalisador desativado usada nos testes foi de aproximadamente $10 \mathrm{mg}$. Após ser estabelecida uma vazão constante de $100 \mathrm{~mL} / \mathrm{min}$ de ar sintético, a temperatura foi elevada até $900^{\circ} \mathrm{C}$ a uma taxa de aquecimento de $10^{\circ} \mathrm{C} / \mathrm{min}$.

\section{RESULTADOS E DISCUSSÃO}

\subsection{Testes Preliminares}

Os testes preliminares foram feitos nas temperaturas de 500 e $550^{\circ} \mathrm{C}$, com tempos de regeneração similares para ambos os ensaios. Os mesmos podem ser visualizados nas Figuras 1a e 1b, que expressa a atividade do catalisador em termos de conversão de metano, bem como a evolução da pressão no reator. 
A análise comparativa destas figuras mostra que a $550^{\circ} \mathrm{C}$ a conversão de metano foi maior, porém o tempo de cada ciclo e o tempo total foi inferior quando comparado com a reação a $500^{\circ} \mathrm{C}$.

É possível perceber também, para ambos os ensaios, que nos dois primeiros ciclos a reação foi interrompida pelo aumento da pressão no reator, ou seja, pelo entupimento do mesmo. Já para os últimos dois ciclos a reação foi interrompida por uma acentuada queda na atividade do catalisador.
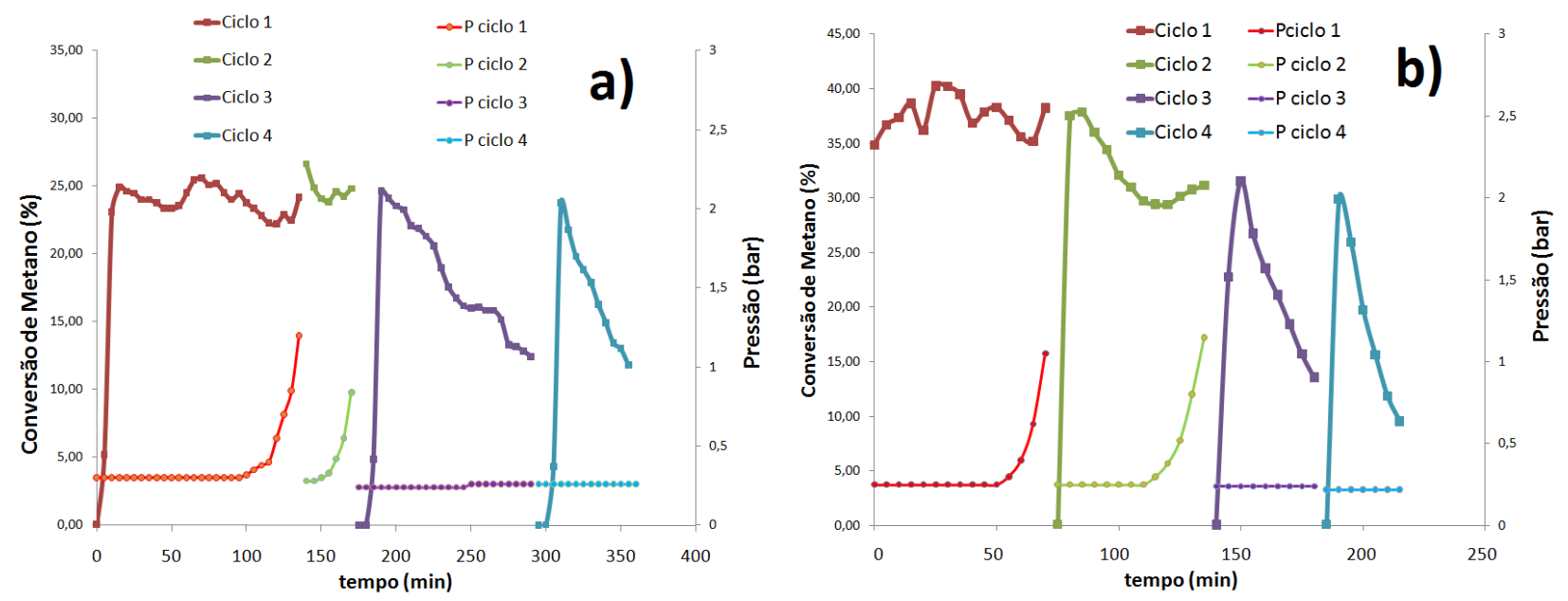

Figura 1 - Conversão de metano e pressão do reator para testes feitos a: a) $500^{\circ} \mathrm{C}$; b) $550^{\circ} \mathrm{C}$.

\subsection{Testes com tempo de regeneração variável}

Os ensaios com diferentes tempos de regeneração podem ser visualizados na Figura 2, É possível observar que, nos testes com 30 e 20 minutos de regeneração, logo após o final de cada ciclo e a seqüente regeneração do catalisador, a atividade do mesmo foi próxima de zero no início do ciclo, necessitando de alguns minutos sob a passagem de metano para que o catalisador pudesse recuperar sua atividade. Este comportamento indica que o tempo de regeneração de 20 minutos ou superior, é excessivo e promove a oxidação do metal cobalto presente no catalisador para a forma de óxido, o qual é novamente reduzido para a forma metálica quando submetido à passagem de metano no reator.

No teste realizado com 10 minutos de regeneração, observa-se que, logo após a passagem de ar, a atividade do catalisador retorna aos níveis anteriores, indicando que o catalisador não sofreu uma oxidação significativa na etapa de regeneração.

De acordo com os resultados obtidos, optou-se por fazer os testes seguintes com tempo de regeneração de 10 minutos. 

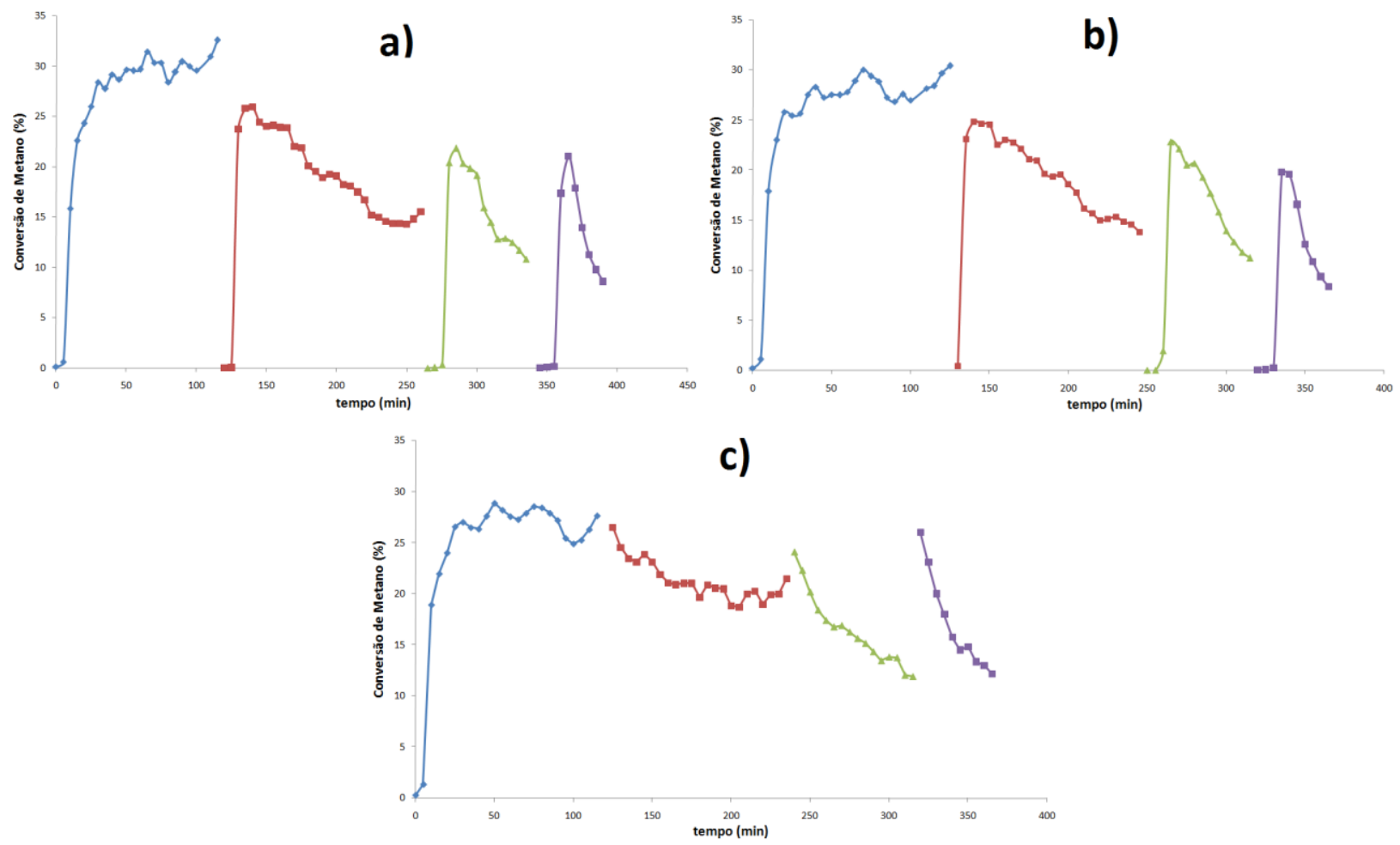

Figura 2 - Conversão de metano para testes feitos com tempo de regeneração de: a) 30 minutos; b) 20 minutos; c) 10 minutos.

\subsection{Testes com diferentes concentrações de oxigênio na regeneração} Tabela 1.

As proporções de oxigênio utilizadas na etapa seguinte dos ensaios encontram-se descritas na

Tabela 1 - Proporção de oxigênio na mistura oxidante utilizada na regeneração do catalisador

\begin{tabular}{c|c|c|c}
\hline $\begin{array}{c}\text { Vazão Ar } \\
(\mathrm{mL} / \mathrm{min})\end{array}$ & $\begin{array}{c}\text { Vazão Nitrogênio } \\
(\mathrm{mL} / \mathrm{min})\end{array}$ & $\begin{array}{c}\text { Vazão total da } \\
\text { mistura }(\mathrm{mL} / \mathrm{min})\end{array}$ & $\begin{array}{c}\text { Vazão Oxigênio } \\
(\mathrm{mL} / \mathrm{min})\end{array}$ \\
\hline 75 & 15 & 90 & 15 \\
50 & 40 & 90 & 10 \\
25 & 65 & 90 & 5 \\
\hline
\end{tabular}

É possível observar na Figura 3a um comportamento similar ao teste feito com 10 minutos de regeneração da etapa anterior, porém com uma conversão levemente inferior no segundo ciclo. 
No teste realizado com $10 \mathrm{~mL} / \mathrm{min}$ de oxigênio (Figura $3 \mathrm{~b}$ ), percebe-se que não ocorreu a oxidação completa do material carbonáceo, o que resultou no entupimento do reator durante os 4 ciclos do teste. Embora a atividade não tenha diminuído acentuadamente, o tempo de campanha do catalisador foi muito curto quando comparado aos testes anteriores.
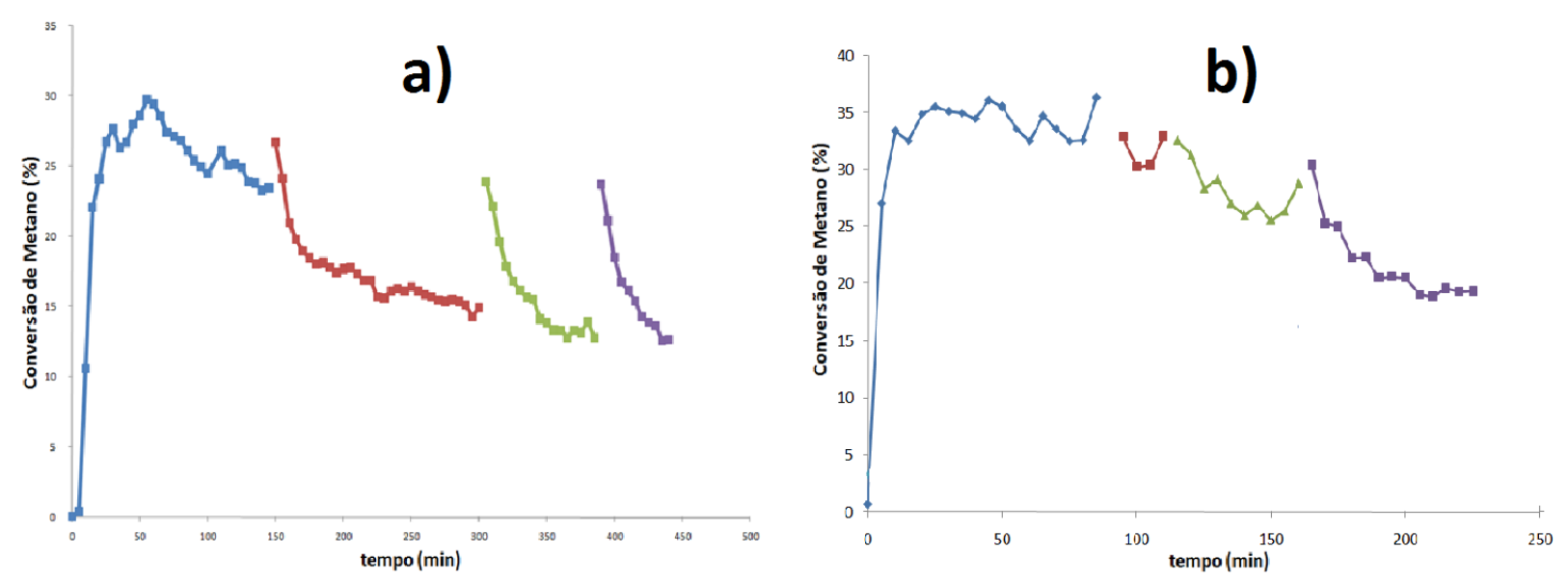

Figura 3 - Conversão de metano com passagem de: a) $15 \mathrm{~mL} / \mathrm{min}$ de oxigênio nas etapas de regeneração do catalisador; b) $10 \mathrm{~mL} / \mathrm{min}$ de oxigênio nas etapas de regeneração do catalisador.

No teste feito com a passagem de $5 \mathrm{~mL} / \mathrm{min}$ de oxigênio na mistura oxidante, observou-se que a oxidação do carbono depositado foi incompleta. Neste caso, observou-se que durante os dez minutos de regeneração, não houve diminuição de pressão no reator, o que impossibilitou a continuidade do teste nessas condições, razão pela qual estes dados não foram apresentados na Figura 3.

De acordo com os resultados obtidos nestes ensaios, a diluição de ar com nitrogênio não ocasionou melhora significativa ao processo de regeneração do catalisador.

\subsection{Análise termogravimétrica do catalisador desativado}

Outra forma de avaliar o tempo adequado de oxidação do carbono depositado é através de análise termogravimétrica. $\mathrm{O}$ resultado da Figura 5 refere-se à amostra submetida a apenas um ciclo de reação sem regeneração. Após a retirada da amostra do reator tubular, $10 \mathrm{mg}$ da mesma foram utilizadas na termobalança. $\mathrm{O}$ ensaio foi conduzido até a temperatura de $500^{\circ} \mathrm{C}$ numa taxa de aquecimento de $10^{\circ} \mathrm{C}$ por minuto sob passagem de $100 \mathrm{~mL} / \mathrm{min}$ de nitrogênio. Após o estabilização da temperatura de $500^{\circ} \mathrm{C}$ procedeu-se a troca de nitrogênio para ar sintético, sendo estas condições mantidas por 1 hora. Os resultados da análise térmica diferencial e da derivada da massa na Figura 5 indicam que a oxidação do carbono depositado é totalmente efetuada num tempo aproximado de 12 minutos, sendo que a taxa máxima de oxidação do carbono ocorre em aproximadamente 9 minutos. 


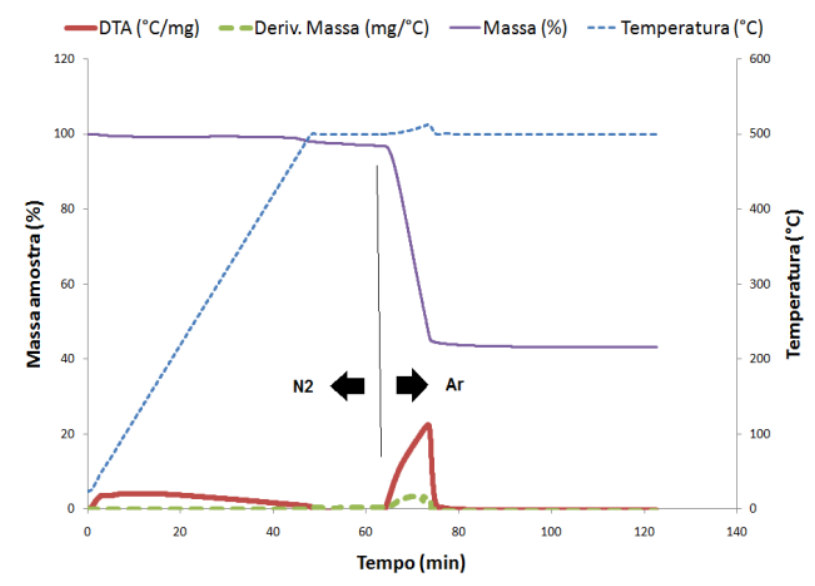

Figura 5 - Análise termogravimétrica de catalisador com carbono depositado.

\subsection{Análise de TPO/DTA do catalisador desativado}

Para a análise do tipo de carbono depositado sobre o catalisador, efetuaram-se análises de TPO/DTA nos catalisadores desativados dos testes feitos sob diferentes concentrações de oxigênio na mistura oxidante. Os resultados mostraram um comportamento semelhante para as amostras. Na Figura 6 pode-se observar os resultados obtidos para o teste feito com 15 e $10 \mathrm{~mL} / \mathrm{min}$ de oxigênio. Observa-se inicialmente um pequeno aumento na massa da amostra devido à oxidação do metal presente. Em temperaturas na faixa de 400 a $450^{\circ} \mathrm{C}$, pode-se observar a oxidação do carbono presente nas amostras, sendo que os picos das análises diferenciais indicaram temperaturas de 431 e $426{ }^{\circ} \mathrm{C}$ para os testes de 15 e $10 \mathrm{~mL} / \mathrm{min}$ de oxigênio respectivamente. A largura do pico principal e o pequeno pico próximo a $470^{\circ} \mathrm{C}$ sugerem filamentos de carbono (Escobar, 2012 e Hermes, 2010), embora haja a necessidade de estudos complementares.
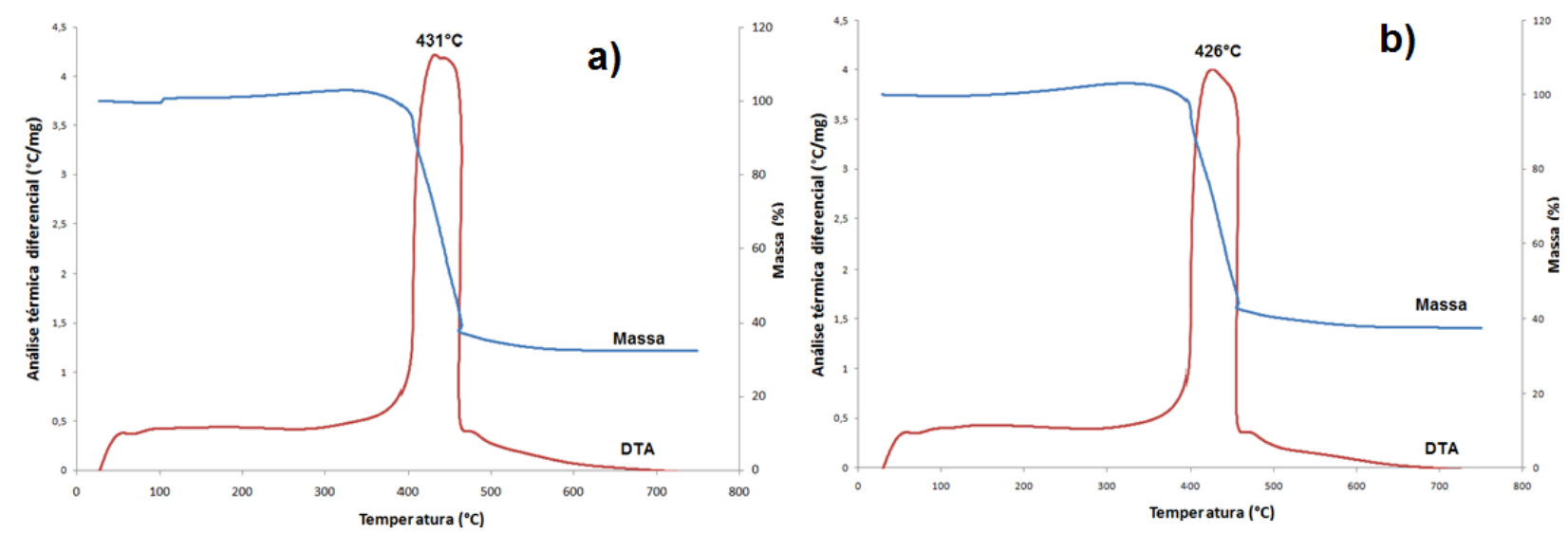

Figura 6 - Análise de TPO/DTA realizada no teste de regeneração com: a) $15 \mathrm{~mL} / \mathrm{min}$ de $\mathrm{O}_{2}$ b) $10 \mathrm{~mL} / \mathrm{min}$ de $\mathrm{O}_{2}$. 


\section{9 a 22 de outubro de 2014 \\ Florianópolis/SC}

\section{CONCLUSÕES}

Através dos testes de atividade dos catalisadores realizados neste estudo, foi possível observar que tempos de regeneração com ar puro entre 10 e 15 minutos são os mais adequados para a produção de hidrogênio nas condições avaliadas. Testes com tempos maiores indicaram uma oxidação acentuada do catalisador e uma conseqüente queda na atividade do mesmo.

A diluição do oxigênio na mistura oxidante não resultou em melhor desempenho do catalisador. Nos testes com 5 e $10 \mathrm{~mL} / \mathrm{min}$ de oxigênio, observou-se a oxidação incompleta do carbono depositado e a diminuição do tempo de duração da reação de decomposição catalítica do metano devido ao rápido entupimento do reator.

Os valores de conversão apresentados nos testes de atividade indicam que, depois da segunda regeneração realizada, a atividade do catalisador cai acentuadamente, indicando sinterização das partículas metálicas.

As análises de TPO realizadas indicam a presença de carbono amorfo, e possivelmente, filamentos de carbono depositados sobre o catalisador.

\section{REFERÊNCIAS}

ARMOR, J. N. The multiple roles for catalysis in the production of $\mathrm{H}_{2}$. Applied Catalysis A: General, V. 176, p. 159-176, 1999.

AVDEEVA, L. B.; KOCHUBEY, D. I.; SHAIKHUTDINOV, S. K. Cobalt catalysts of methane decomposition: accumulation of the filamentous carbon. Applied Catalysis A: General, v. 177, p. 43-51, 1999.

ESCOBAR, C. C. Decomposição do metano sobre catalisadores Co-Al modificados com cobre. Dissertação (Mestrado em Engenharia Química). Universidade Federal do Rio Grande do Sul, 2012.

HERMES, N. A. Hidrogênio e nanotubos de carbono por decomposição catalítica do metano: desempenho de catalisadores à base de cobalto e alumínio. Dissertação (Mestrado em Engenharia Química). Universidade Federal do Rio Grande do Sul, 2012.

HOLLADAY, J. D.; HU, J.; KING, D. L.; WANG, Y. An overview of hydrogen production Technologies. Catalysis Today, v. 139, p. 244-260, 2009.

KONIECZNY, A.; MONDAL, K.; WILTOWSKI, T.; DYDO, P. Catalyst development for thermocatalytic decomposition of methane to hydrogen. International Journal of Hydrogen Energy, $\mathrm{v}$. 33, p. 264-272, 2008.

PEREZ-LOPEZ, O. W.; SENGER, A. Estudo da produção de hidrogênio de elevada pureza a partir da decomposição catalítica do metano. In: XV Congresso Brasileiro de Engenharia Química, Curitiba, Paraná, Anais, 2004.

PÉREZ-RAMÍREZ, J., MUL, G. On the stability of the thermally decomposed Co-Al hydrotalcite against retrotopotactic transformation. Materials Research Bulletin, v.36, p. 1767-1775, 2001. 